\title{
Capacity Enhancement with Joint Subchannel and Power Allocation Scheme for OFDMA Femtocell Networks
}

\author{
Arathy Varghese \\ Electronics and Communication Engineering \\ NSS College of Engineering \\ Palakkad, Kerala, India
}

\author{
Sudha T \\ Electronics and Communication Engineering \\ NSS College of Engineering \\ Palakkad, Kerala, India
}

\begin{abstract}
Efficient power and subchannel allocation methods are required for orthogonal frequency division multiple access (OFDMA) based femtocell networks to improve the capacity of the system. This paper considers a joint subchannel and power allocation algorithm with capacity maximization for downlink of an OFDMA based femtocell networks. In the proposed algorithm subchannel allocation is first performed based on signal to interference plus noise ratio (SINR) of the channel with equal power distribution. Then for enhancing capacity with optimal power allocation, successive convex approximation (SCA) based power optimization is adopted. The effect of Arithmetic geometric mean (AGM) approximation with SCA on power optimization is also investigated. The optimal power is subsequently distributed by water-filling algorithm.
\end{abstract}

\section{Keywords}

Capacity enhancement,Convex optimization, Femtocell, Power allocation, SINR, Subchannel assignment, Successive convex approximation

\section{INTRODUCTION}

One of the major challenges for next generation wireless communication systems is to improve the indoor coverage and provide high data rate services to the users in a cost effective manner and at the same time, to enhance network capacity [1]. Since $70 \%$ of data traffic and $50 \%$ of voice traffic are orginated from the mobile users in the indoor regions, serving those users by indoor systems is necessary which will effectively extend the coverage of mobile communication systems with low cost. The surest way to increase the system capacity of a wireless link is by getting the transmitter and receiver closer to each other, which creates the dual benefits of higher quality links and more spatial reuse.

Femtocells are the cost effective alternate solution for the above problem. Femtocells or home base stations are data access points which are installed by home users for getting high data services to indoor regions. Femtocells or Femto Access Points (FAPs) are small, short-ranged $(10-30 \mathrm{~m})$ low powered $(10-100 \mathrm{~mW})$ access points developed to provide cost effective and high-bandwidth services in next generation wireless communication systems. One of the efficient method for multiple access is orthogonal frequency division multiple access(OFDMA) 2] which is used in many of the current communication standards. It is also a strong candidate for next generation cellular systems. The most important feature of OFDMA systems is that it can incorporate dynamic RA, which allocates subcarriers, power, and bits to multiple users when the transmitter has the channel state information to all users available. So the concept of OFDMA based femtocell network exploit the benefits of both. Thus an efficient subcarrier and power allocation will improve the performance or throughput of the system.

In OFDMA-based femtocell networks, due to flexibility for dynamic RA orthogonal sub-carriers can be assigned to femtocells and macrocells and OFDMA has been used as the air interface technology by Long Term Evolution (LTE) femtocells, i.e., home evolved Node Bs (HeNBs). The co-tier interference of the network is completely avoided with the use of OFDMA scheme [3]. Nonetheless, cochannel deployment implies that an OFDM subchannel can be shared by UEs from different cells, giving rise to the intercell interference. Further more, there is another source of technical difficulty is that the subchannel assignment problem which involves the allocation of the limited radio frequencies to different UEs in multiple cells. To directly solve this combinatorial problem, approaches with an exponential complexity are required. Therefore, the successful development of any resource allocation scheme for OFDMA based femtocell networks certainly relies upon how one can effectively overcome such a difficult problem. In wireless cellular or ad hoc networks where a variety of subchannel allocation and power control problems can be formulated as optimization problems with a system wide objective, e.g., maximizing the total system capacity, subject to QoS constraints ,e.g., on data rate.

The work in [4] solves the resource allocation problem for multicell OFDMA-based network using a noncooperative game theory approach. The study in [5] considers game approach to solve the problem of joint power allocation and subchannel assignment in the downlink of a multicell OFDMA network. Another scheme is an improved iterative waterfilling algorithm, adapted to this multicell scenario. It is noted that all the solutions developed in [6] depend on a central unit to collect and process the complete channel state information. To alleviate the high complexity required by such solutions, [7 proposes a distributed low complexity scheme based on the concept of a reference user. Considering the downlink of an OFDMA network, [8] addresses the problem of maximizing the weighted sum of the minimal UE rates of coordinated cells. A 
centralized algorithm proposed by [8] alternatively optimizes the subchannel assignment and power allocation. At each iteration, the allotment of subchannels is updated by resolving a mixed integer linear program for each cell. The optimal allocation of powers is found by a duality based numerical algorithm. However, if a minimum rate constraint is strictly imposed so as to guarantee the QoS of some UEs, it can be shown that the solutions developed in [6] 8] are no longer applicable.

A power allocation algorithm called SCALE, which is similar to the SCA mthod was introduced in [9].The method results in higher achievable rates than the competitive optimal rates resulting from simultaneous water-filling. Message-passing overhead in communication can be limited through the use of this algorithm. SCALE can perform better than IWF. SCALE can converge to rates satisfying the necessary KKT [10] optimality condition far exceeding that of IWF with just two to three iterations, and to within $90 \%$ of the final rate in under five iterates. But it is not solving a discrete optimization problem.

Capacity maximization can be achieved by a technique in [11] for dense environments of cells. It proposes a centralized algorithm for joint power and subchannel allocation scheme in which power allocation is done by BPA method which has less computational complexity. But the above algorithm doesn't built on a multiple access scheme and femtocell based networks. Where the work in [12] presented two-tier networks that employ OFDMA and propose a decentralized spectrum allocation scheme based on area spectral efficiency (ASE) subjected to a quality of service (QoS) requirement, which guarantees that both macrocell and femtocell users attain atleast a prescribed data rate. Another technique in [13] has the capacity improvement for both delay sensitive users and delay tolerant users. The resource allocation problem is modeled as a mixed integer programming problem and then, it is transformed into a convex optimization problem and is a low-complexity resource allocation algorithm. Here the optimization considered is not a joint optimization technique.

In [14] subchannel and power allocation is done by the process of optimization through the use of some iterative algorithm. In this [14] power optimization was done using three approximation techniques which are based on SCA method. Among these three, AGM approximation based on SCA is a centralized algorithm which is used to maximize the system throughput. But the system lacks of some capacity enhancement technique. Two methods for capacity enhancement is given in [15] with the resource allocation for MU-OFDMA network scenario. The optimization problem considers maximizing the sum capacity while maintaining proportional fairness among users for each channel realization. The algorithm to find the optimal solution is discussed which is a low complexity suboptimal algorithm. The algorithm to find the optimal solution is discussed which is a low complexity suboptimal algorithm. The optimal power distribution is a two step procedure. First, non-convex optimization has to be done in order to get the power distribution among users. Then to a particular user, the greedy waterfilling algorithm is adopted to maximize the capacity.

In this paper, we have formulated an algorithm to enhance the capacity of OFDMA femtocell network with the AGM approximation based on SCA optimization technique. The algorithm balances a trade-off between capacity and fairness. Subchannel allocation has been done first using the channel quality parameters and then power optimization is done by SCA method and also by AGM approximation based on SCA. After power optimization, an algorithm checks the gain of the selected channel. In some cases the selected channel does not have the required quality, which may cause the wastage of the same. Hence as the next step the channel parame-

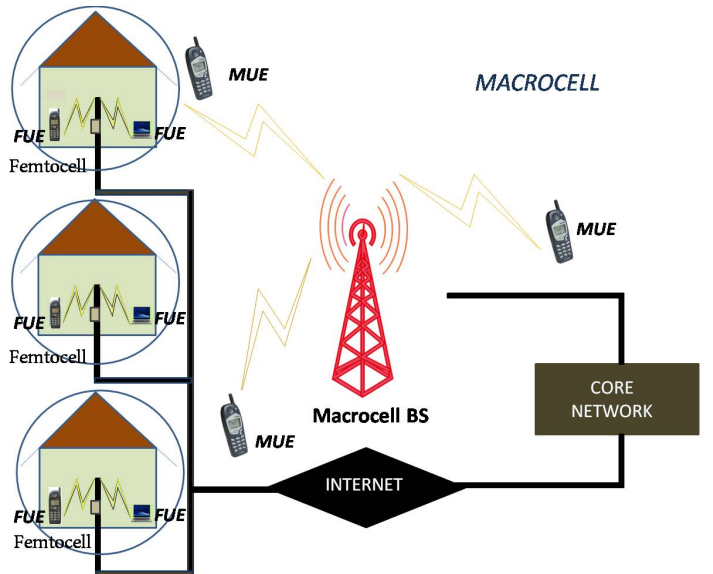

Fig. 1. OFDMA based heterogeneous network with one macrocell and several femtocell

ters are updated to retrieve the quality of the channel and to ensure sufficient gain thereby minimizing the wastage of channels. Then optimal power is distributed to every single user by means of waterfilling algorithm to maximize capacity.

The paper is organized as follows, section 2 gives the system model of OFDMA femtocell networks with the power constraints. Section 3 describes the proposed system. Section 4 discuss about the simulation results. Section 5 briefs the conclusions derived in this paper.

\section{SYSTEM MODEL}

Consider a two tier femtocell/macrocell network environment with macrocell surrounded with several femtocell as shown in figure(1). Authors assume that the OFDMA system is having a bandwidth W $\mathrm{Hz}$ which is divided into equal $\mathrm{K}$ subchannels of $\mathrm{B} \mathrm{Hz}$ each. Let $\mathrm{X}$ be the total number of FAP. Each femtocell has atleast one FUE under its control. All the FAPs are assumed to be located in indoor residential areas. During the process of optimizing power and subchannel, it is assumed that the association of particular UE with its own BS is fixed. Let $U$ be the total number of users of the OFDMA system with transmit power $P_{x}(k)$ for the femtocell $x$ in subchannel $k$. Denote $P(k)=\left[p_{0}(k), p_{1}(k), \ldots p_{X}(k)\right]^{T}$, $P_{x}=\left[p_{x}(1), p_{x}(2), \ldots p_{x}(k)\right]^{T}$ and $P=\operatorname{vec}\left[P_{0}, P_{1}, \ldots, P_{X}\right]$ The received SINR at UE $u \in U$ on subchannel $k \in K$ is expressed as:

$$
S_{x, u}(P(k))=\frac{p_{x}(k) h_{x, u}(k) g_{\alpha}(x-u)}{\sum_{y \in \phi(x)} p_{y}(k) h_{y, u}(k) g_{\alpha}(y-u)}
$$

where $\phi(x)$ is the set of transmitters in $\phi$ that interferes with $\mathrm{x}, P_{y}$ is the power of the transmitting node at $\mathrm{y}$, and $h_{x, u}$ is the channel gain from transmitter $\mathrm{x}$ to receiver $\mathrm{u}$. Noise is ignored since authors focus on the interference-limited regime. $g_{\alpha}(z)=\|z\|^{-\alpha}$ is the standard power law path loss function, where $\alpha$ is the path loss exponent and $\|$.$\| is the Euclidean norm. Here consider \alpha_{i}$ as the pathloss component of indoor-indoor link and $\alpha_{o}$ as the pathloss component of outdoor-outdoor link. For the outdoor-outdoor link the distance D can be considered as the distance between MBS and MUE. But for indoor-indoor link D can be considered as the distance between FAP and FUE. Pathloss exponent $\alpha$ can be derived from the distance between transmitter $\mathrm{x}$ and UE $\mathrm{u}$ which is calcu- 
lated as,

$$
D=\sqrt{A^{2}+B^{2}}
$$

where $\mathrm{A}$ and $\mathrm{B}$ are the distance from transmitter to receiver from the points $\mathrm{a}$ and $\mathrm{b}$ respectively. From the Eq. 2, pathloss exponent $\alpha$ is defined by,

$$
\alpha=10^{-\alpha^{\prime} / 10}
$$

$$
\text { where } \alpha^{\prime}=128.1+37.6 \log _{10}
$$

The instantaneous data rate attained by UE $u \in U$ on subchannel $k \in K$ can be written as:

$$
r_{x, u}(P(k))=\ln \left(1+S_{x, u}(P(k))\right)
$$

subchannel assignment can be represented by $\rho_{x, u}(k)$ where $\rho_{x, u}(k)=1$ if $k \in K$ is assigned to $u \in U$ and $\rho_{x, u}(k)=0$ otherwise. Denote $\rho_{x, u}=\left[\rho_{x, u}(1), \rho_{x, u}(2), \ldots \rho_{x, u}(K)\right]^{T}, \rho_{x}=$ $\operatorname{vec}\left[\rho_{x, 1}, \rho_{x, 2}, \ldots \rho_{x, U}\right]^{T}$ and $\rho=\operatorname{vec}\left[\rho_{0}, \rho_{1}, \ldots, \rho_{X}\right]$. The total throughput attained by UE $u \in U$ can be computed as:

$$
T_{x, u}\left(\rho_{x, u}, P\right)=\sum_{k \in K} \rho_{x, u}(k) r_{x, u}(k)(P(k))
$$

It is to be noted that in a heterogeneous network lower tier femtocell is targeting to maximize the throughput with the consideration of macrocell priority. Thus the resource management problem can be formulated as follows.

$$
\begin{gathered}
\max _{P, \rho} \sum_{x \in X} \sum_{u \in U} \sum_{k \in K} \rho_{x, u}(k) r_{x, u}(P(k)) \\
\text { subject to } \sum_{u \in U} \rho_{x, u}(k)=1, \forall x \in X, \forall k \in K \\
\rho_{x, u}(k) \in\{0,1\}, \forall x \in X, \forall k \in K, \forall u \in U \\
\sum_{k \in K} p_{x}(k) \leq P_{x}^{\max }, \forall x \in X
\end{gathered}
$$

Equation (6) represents the total throughput of all femtocells, the orthogonal assignment of subchannels with the assumption of OFDMA and shows that transmit powers are limited by total power limit. The aim is to optimize power and subchannel allocation for both MUE and FUE with the objective of maximizing the total throughput and capacity of the system and protecting minimum capacity for the prioritized macrocell.

\section{JOINT SUBCHANNEL AND POWER ALLOCATION}

To solve the resource management problem in equation 6 the following iterative approach of joint subchannel and power allocation is employed.

\subsection{Initial Power Allocation}

To find a feasible solution $(\rho, P)$ for power and subchannel allocation problem, the difficulty lies in how one meets the macrocell sum rate constraint. Macrocell BS is indicated by 0 and, $S_{0, u}$ is the SINR attained by MUE. If $R_{\min }$ is greater than the maximum throughput achievable by the macrocell, it is obvious that the optimal solution is infeasible. To determine the highest attainable macrocell throughput $R *$, It is assumed that all femtocell BSs do not transmit (i.e., $P_{x}=0, \forall x \in X$ ), and that each OFDM subchannel is assigned to the MUE with the highest SINR on that subchannel. The following equations describes the optimization problem for the macrocell.

$$
\max _{P_{0}} \sum_{u \in U} \sum_{k \in K} \rho_{0, u}(k) r_{0, u}(P(k))
$$

$$
\text { subject to } \sum_{k \in K} p_{0}(k) \leq P_{0}^{\max }, \forall x \in X
$$

\subsection{Subchannel Allocation}

For a given initial power, authors try to find optimal subchannel assignment $\rho$ for each UE belongs to a specific FAP. Good channels can be selected by the SINR value of the channel. Since no FAP is transmitting initially, FUEs do not need any channel for data transmission then the subchannel is allocated only for MUEs. So each MUE can associate the channels with higher data rates. There by gives priority to the MUEs. The optimum subchannel allocation can be formulated as,

$$
\begin{gathered}
\max _{\rho} \sum_{x \in X} \sum_{u \in U} \sum_{k \in K} \rho_{x, u}(k) r_{x, u}(P(k)[t-1]) \\
\text { subject to } \sum_{u \in U} \sum_{k \in K} \rho_{0, u}(k) r_{0, u}(P(k)[t-1]) \geq R_{\text {min }} \\
\sum_{u \in U} \rho_{x, u}(k)=1, \forall x \in X, \forall k \in K \\
\rho_{x, u}(k) \in\{0,1\}, \forall x \in X, \forall k \in K, \forall u \in U
\end{gathered}
$$

For solving the subchannel allocation problem in equation 8 a subchannel is allotted to MUES after a long search for channels with highest data rate(highest SINR). Then subchannel assignment is to be done for each FUEs. It is easy to assign subchannels for FUEs because an exhaustive search for channels is already completed for finding the optimal solution for equation 8 .

\subsection{Power Allocation with Fixed Subchannel Assignment}

Assuming that the subchannel allocation $\rho$ is given the optimum power allocation problem can be formulated as,

$$
\max _{P} \sum_{x \in X} \sum_{k \in K} r_{x, u}(P(k))
$$

$$
\text { subject to } \sum_{k \in K} p_{x}(k) \leq P_{x}^{\max }, \forall x \in X
$$

The problem in equation 9 is not convex because of the concave nature of the function. This work adopt the SCA approach for finding the optimum power allocation. Apart from SCA method, authors are also investigating another optimization solution based on SCA approach known as AGM approximation. The steps of SCA method are explained below. Optimal power allocation find in the time scale $t_{p}$.

(1) Set a feasible point at $\mathrm{P}[0]$ for initialization. 
(2) Form a $t_{p}^{t h}$ convex subproblem by approximating the non concave objective function and constraints

(3) Solve the above formulated convex sub problem to obtain optimal solution $P\left[t_{p}\right]$.

(4) Update the parameters after optimization.

(5) Increment the point of optimization $t_{p}=t_{p}+1$, go back to Step 2 until the value of power at that point converges to $P\left[t_{p}\right]$.

3.3.1 Centralized SCA based power allocation with AGM Approximation. The SCA approach converges to a locally optimum solution that satisfies Karush-Kuhn-Tucker (KKT)[10] conditions with AGM approximation [equation[9]]. To solve the step 2 and step 4 of the SCA approach, AGM approximation is used. To solve for power constraints in equation 9), AGM approximation algorithm works as explained in Table 1 .

The problem in equation(9) can also be written as,

$$
\begin{gathered}
\min P \prod_{x \in X / 0} \prod_{k \in K} \frac{\sum_{y \in \phi(x) / x} p_{y}(k) h_{y, u}(k) g_{\alpha}(y-u)}{\sum_{x \in X} p_{x}(k) h_{x, u}(k) g_{\alpha}(x-u)} \\
\text { s.t } \prod_{k \in K} \frac{\sum_{y \in \phi(x) / 0} p_{y}(k) h_{y, u}(k) g_{\alpha}(y-u)}{\sum_{x \in X} p_{0}(k) h_{0, u}(k) g_{\alpha}(0-u)} \leq \exp \left(-R_{\min }\right) \\
\sum_{k \in K} p_{x}(k) \leq P_{x}^{\max }, \quad \forall x \in X
\end{gathered}
$$

If we define,

$$
f_{x}(k)\left(p_{x}(k)\right)=p_{x}(k) h_{x, u}(k) g_{\alpha}(x-u)
$$

The algorithm for AGM approximation compute the monomials of the objective function. A geometric program like equation(12) can easily be transformed into a convex problem using monomial via a logarithmic change of variables.

$$
\begin{gathered}
\min P \prod_{x \in X / 0} \prod_{k \in K} \frac{\sum_{y \in \phi(x) / x} p_{y}(k) h_{y, u}(k) g_{\alpha}(y-u)}{w_{x}(k)(p(k))} \\
\text { s.t } \prod_{k \in K} \frac{\sum_{y \in \phi(x) / 0} p_{y}(k) h_{y, u}(k) g_{\alpha}(y-u)}{w_{x}(k)(p(k))} \leq \exp \left(-R_{\min }\right) \\
\sum_{k \in K} p_{x}(k) \leq P_{x}^{\max }, \forall x \in X
\end{gathered}
$$

\subsection{Optimal Power Allocation Through Power Distribution Among Multiple User}

In this section, the optimal power distribution strategy for a multiple users is given. Total power will be distributed to the channel according to the capacity constraints as,

$$
\begin{gathered}
\sum_{x \in X}\left(\log _{2}\left(1+S_{x, 1}(k) \frac{p_{x, 1}(k)-V_{x, 1}}{K_{1}}\right)+\log _{2} Y_{x, 1}\right)= \\
\sum_{x \in X}\left(\log _{2}\left(1+S_{x, u}(k) \frac{p_{x, u}(k)-V_{x, u}}{K_{u}}\right)+\log _{2} Y_{x, u}\right)
\end{gathered}
$$

Where $p_{x, u}$ is the power distributed to UE u under the control of a particular FAP x where $u \in U$ and $x \in X . \forall u=2,3, \ldots, U$ where
Table 1. Centralized SCA-based Power Allocation with AGM Approximation

1. Set a feasible point $t_{p}=1$.

2. Repeat the following steps to solve for equation 9

3. Compute each term $f_{x}(k)\left(p_{x}(k)\left[t_{p}-1\right]\right), \forall x \in X, \forall k \in K$ in equation 10 .

4. Compute each coefficient.

$$
v_{x}(k)\left[t_{p}\right]=\frac{f_{x}(k)\left(p_{x}(k)\left[t_{p}-1\right]\right)}{\sum_{y \in Y} f_{y}(k)\left(p_{y}(k)\left[t_{p}-1\right]\right)}, \forall x \in X, \forall k \in K
$$

5. Compute monomial.

$$
w_{x}(k)\left[t_{p}\right](P(k))=\prod_{x \in X}\left(\frac{f_{x}(k)\left(p_{x}(k)\left[t_{p}-1\right]\right)}{v_{x}(k)\left[t_{p}\right]}\right)^{v_{x}(k)\left[t_{p}\right]}
$$

6. With $w_{x}(k)\left[t_{p}\right](P(k))$ solve (convex) geometric program equation 10

7. Set $t_{p}=t_{p}+1$.

8. until Convergence of $\mathrm{p}$ and $\rho$

$V_{x, u}$ and $Y_{x, u}$ are defined as,

$$
\begin{gathered}
V_{x, u}=\sum_{k=2}^{K} \frac{S_{x, u}(k)-S_{x, u}(1)}{S_{x, u}(k) S_{x, u}(1)} \\
Y_{x, u}=\left(\prod_{k=1}^{K} \frac{S_{x, u}(k)}{S_{x, u}(1)}\right)^{\frac{1}{K}}
\end{gathered}
$$

Adding the total power constraints gives as below

$$
\sum_{u \in U} p_{x, u}=P_{x}^{\max }
$$

There are $\mathrm{U}$ variables $p_{x}(u)_{u=1}^{U}$ in the set of $\mathrm{U}$ equations from equation (13) and equation (16). Solving the set of functions provides the optimal power allocation scheme. The equations are, in general, nonlinear and non-convex in nature. For solving these nonlinear and nonconvex power allocation problems AGM approximation based on SCA can be applied. Optimal power is allocated to subchannel using SCA method only and AGM approximation based on SCA approach.

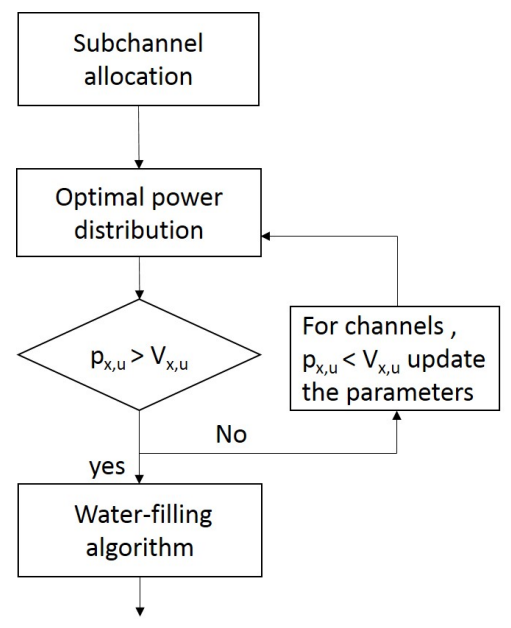

Fig. 2. Proposed algorithm

Figure 2 shows the proposed scheme of capacity enhancement 
with optimum power allocation. System capacity is maximized in the power allocation part. First subchennel allocation is done with equal power distribution for all users under FAPs. Then power is distributed among the users with power optimization algorithm of AGM approximation based on SCA and with SCA alone. The condition $p_{x, u}>V_{x, u}$ should be satisfied in order to maintain the SINR of the channel otherwise channels and the power allotted to the them are wasted because the greedy waterfilling algorithm which distribute optimal power to every user will discard the channel due to low SINR conditions.

\section{SIMULATION RESULTS}

Figure 3 depicts the network scenario for simulation. In the figure MUEs and FUEs are randomly distributed inside the cell of radius $400 \mathrm{~m}$ and $40 \mathrm{~m}$ respectively. Number of MUEs and FUEs are assumed to be $\mathrm{M}=5$ and $\mathrm{U}=20$ respectively. FUEs are equally divided among 4 femtocells. It is to be assumed that the downlink OFDMA transmission is taking place with $\mathrm{K}=16$ subchannels each having bandwidth of $200 \mathrm{KHz}$. The default value of $P_{x}^{\max }=43 \mathrm{dBm}$ for macrocell BS and $P_{x}^{\max }=23 \mathrm{dBm}$ for femtocell BS. Pathloss exponent derived from the equation 3 is having the value of 4 .

Here, authors compare the results of capacity obtained by the

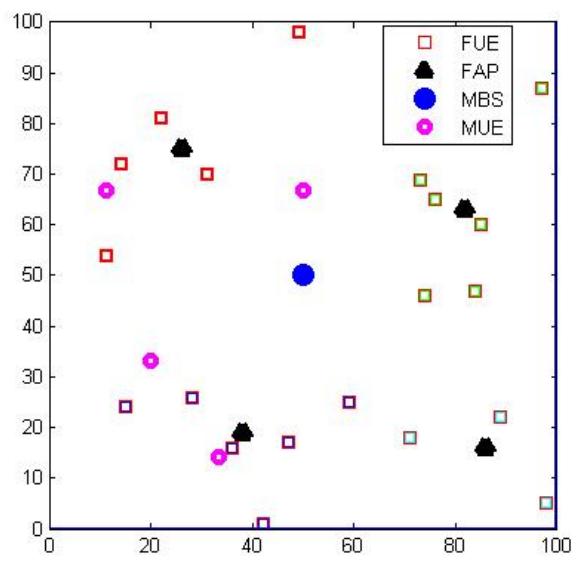

Fig. 3. Network topology in the numerical examples

proposed algorithm with that of the existing algorithm. In order to have fair comparison with the method in [15], in simulations it is modified that the power allocation proposed in [14] with the aim of maximizing the sum capacity of the two- tier femtocell network under consideration. Figure (4) depicts the capacity of the femtocell OFDMA network. In each of these results, the number of femtocells was varied from 16 to 30 in increments of 2, and the capacity obtained in each case was plotted against the number of femtocells.

Figure (4) depicts the total capacity of the existing system is compared with the proposed system. The existing system employs joint subchannel and power allocation with AGM approximation based on SCA method for power optimization. The total capacity of the existing system compared with the proposed system of power allocation with SCA method only and proposed system of power allocation with AGM approximation based on SCA method have been plotted. In both proposed methods, capacity maximization algorithm are incorporated.

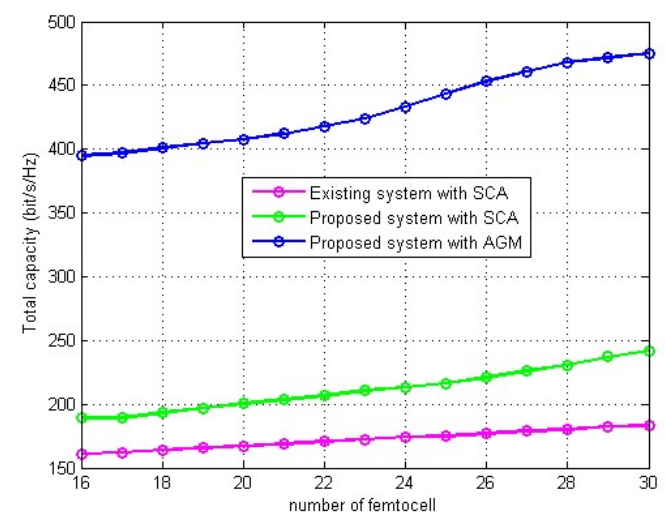

Fig. 4. Total capacity of users in the OFDMA system versus number of femtocells

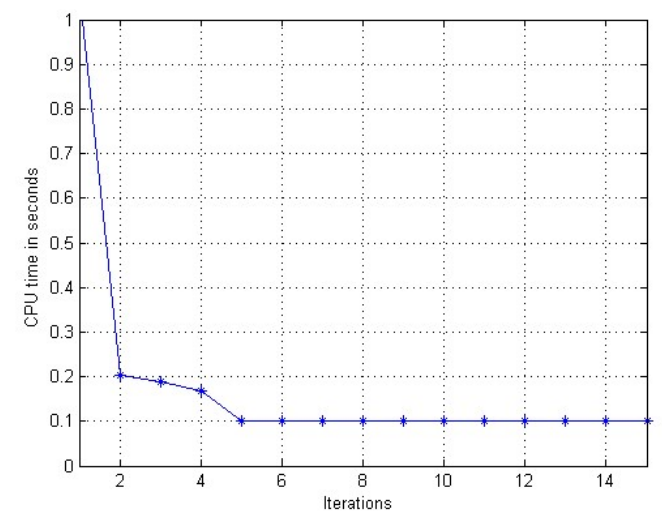

Fig. 5. CPU time

As the number of femtocells increases from 16 to 30 , the system capacity provided by the existing algorithm increases. On the other hand, in the proposed algorithm, system capacity increases even better with AGM approximation based SCA approach than that with SCA method only. Furthermore, it can be observed in figure (4) that the proposed algorithms always provides better capacity as compared with the existing algorithm.

Figure(5) shows the computational time to solve the subchannel and power allocation algorithm. Computational time can also be referred to as CPU time of the femtocells. Less computational time reduces the complexity of the system. The time taken for execution of power and subchannel allocation decreases with increase in the number of iterations. In figure $(5)$ the $\mathrm{CPU}$ time in seconds decreases as the number of iterations increases.

\section{CONCLUSION}

An algorithm for joint subchannel allocation and power optimization which maximizes system capacity in OFDMA based macrocell/ femtocell mixed network is proposed in this paper. Optimal subchannel allocation in each cell based on the SINR value of the particular channel is implemented first. For the channel selected, optimal power has been calculated using SCA based power optimization solutions. Then the optimal power is distributed over 
multiple users. SCA method with a centralized AGM approximation gives better performance on optimization and thus on capacity. Then after checking the channel conditions greedy water-filling algorithm is used distribute the power to every single user to maximize the capacity. It is seen that ergodic capacity of the system is increased much compared to the existing technique and also the capacity variation with number of femtocell users is also improved.

This work doesn't have any technique for cross-tier interference mitigation. Also subchannel allocation is done by a long search method considering only SINR as constraint. So, the future work can be extended for cross-tier interference avoidance with capacity maximization and the method for optimal subchannel allocation can be replaced by some efficient techniques subject to more number of constraints.

\section{ACKNOWLEDGEMENT}

This work is supported by Center for Engineering Research and Development (CERD), Govt. of Kerala, India under the project Research Seed Money (RSM) No. C1/RSM 99/2014

\section{REFERENCES}

[1] Saquib, N.; Hossain, E. Long Bao Le and Dong In Kim, "Interference management in OFDMA femtocell networks: issues and approaches," IEEE Wireless Commununications, vol. 19, pp. 86-95, 2012.

[2] Chandrasekhar, V., Andrews, J.G., Gatherer and Alan, "Femtocell networks: a survey," IEEE Commununication Magazine, vol. 46, pp. 59-67, 2008.

[3] Lopez-Perez, D. Guvenc, I., de la Roche, G. Kountouris, M. Quek, T.Q.S. Jie Zhang, "Enhanced intercell interference coordination challenges in heterogeneous networks," IEEE Wireless Commununication Magazine, vol. 18, pp. 22-30, 2011.

[4] Z. Han, Z. Ji, and K. Liu, "Non-cooperative resource competition game by virtual referee in multi-cell OFDMA networks," IEEE Journal on Selected Areas of Communication, vol. 25, no. 6, pp. 1079-1090, Aug. 2007.

[5] H. Kwon and B. G. Lee, "Distributed resource allocation through noncooperative game approach in multi-cell OFDMA systems," in Proceedings of IEEE International Conference of Communication (ICC), vol. 9, pp. 4345-4350, Jun. 2006.

[6] L. Venturino, N. Prasad, and X. Wang, "Coordinated scheduling and power allocation in downlink multicell OFDMA networks," IEEE Transactions on Vehicular Technology, vol. 58, no. 6, pp. 2835-2848, Jul. 2009.

[7] K. Son, S. Lee, Y. Yi, and S. Chong, "REFIM: A practical interference management in heterogeneous wireless access networks," IEEE Journal on Selected Areas of Communication, vol. 29, no. 6, pp. 1260-1272, Jun. 2011.

[8] T. Wang and L. Vandendorpe, "Iterative resource allocation for maximizing weighted sum min-rate in downlink cellular OFDMA systems," IEEE Transactions on Signal Processing, vol. 59, no. 1, pp. 223-234, Jan. 2011.

[9] J. Papandriopoulos and J. S. Evans, "SCALE: a lowcomplexity distributed protocol for spectrum balancing in multiuser DSL networks," IEEE Transactions on Information Theory, vol. 55, no. 8, pp. 3711-3724, August 2009.

[10] M. Chiang, C. W. Tan, D. P. Palomar, D. ONeill, and D. Julian, "Power control by geometric programming, "IEEE
Transactions on Wireless Communications, vol. 6, no. 7, pp. 2640-2651, July 2007.

[11] J. Kim and D.-H. Cho, "A joint power and subchannel allocation scheme maximizing sys- tem capacity in indoor dense mobile communication systems," IEEE Transactions on Vehicular Technology, vol. 59, no. 9, pp. 4340-4353, Nov. 2010.

[12] Chandrasekhar V. and Andrews J.G., "Spectrum allocation in tiered cellular networks," IEEE Transactions on Communications, vol.57, no.10, pp.3059-3068, October 2009

[13] Haijun Zhang, Chunxiao Jiang, Beaulieu, N.C., Xiaoli Chu, Xiangming Wen, Meixia Tao, "Resource Allocation in Spectrum-Sharing OFDMA Femtocells With Heterogeneous Services,'IEEE Transactions on Communications, vol.62, no.7, pp.2366-2377, July 2014.

[14] Duy Trong Ngo, "Joint Subchannel Assignment and Power Allocation for OFDMA Femtocell Networks, "IEEE Transactions on Wireless Communications, vol. 13, pp.342-355, January 2014.

[15] Zukang Shen, Andrews J.G. and Evans B.L., "Adaptive resource allocation in multiuser OFDM systems with proportional rate constraints," IEEE Transactions on Wireless Communications, vol.4, no.6, pp.2726-2737, Nov. 2005. 